\title{
Vesicle Array-Templated Large-Area Silica Surface Patterns
}

\author{
Qihui Shi, ${ }^{\dagger}$ Jianfang Wang, ${ }^{\dagger}$ Michael D. Wyrsta, ${ }^{*}{ }^{*}$ and Galen D. Stucky ${ }^{*}{ }^{\dagger}$ \\ Department of Chemistry and Biochemistry, University of California, Santa Barbara, \\ California 93106, and Kiowan, Inc., Santa Barbara, CA 93103 \\ E-mail: stucky@chem.ucsb.edu; mdw@kiowan.com
}
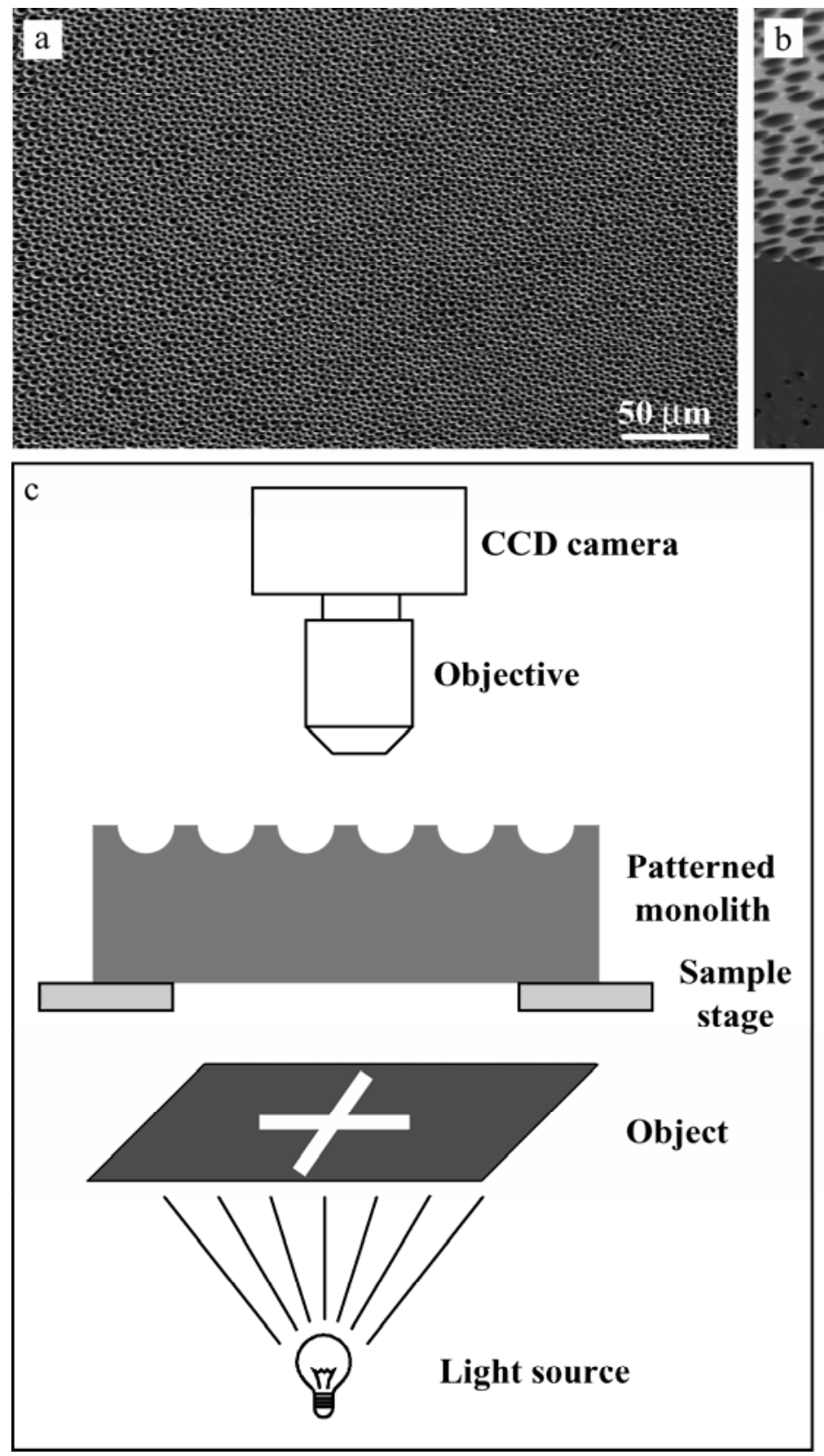
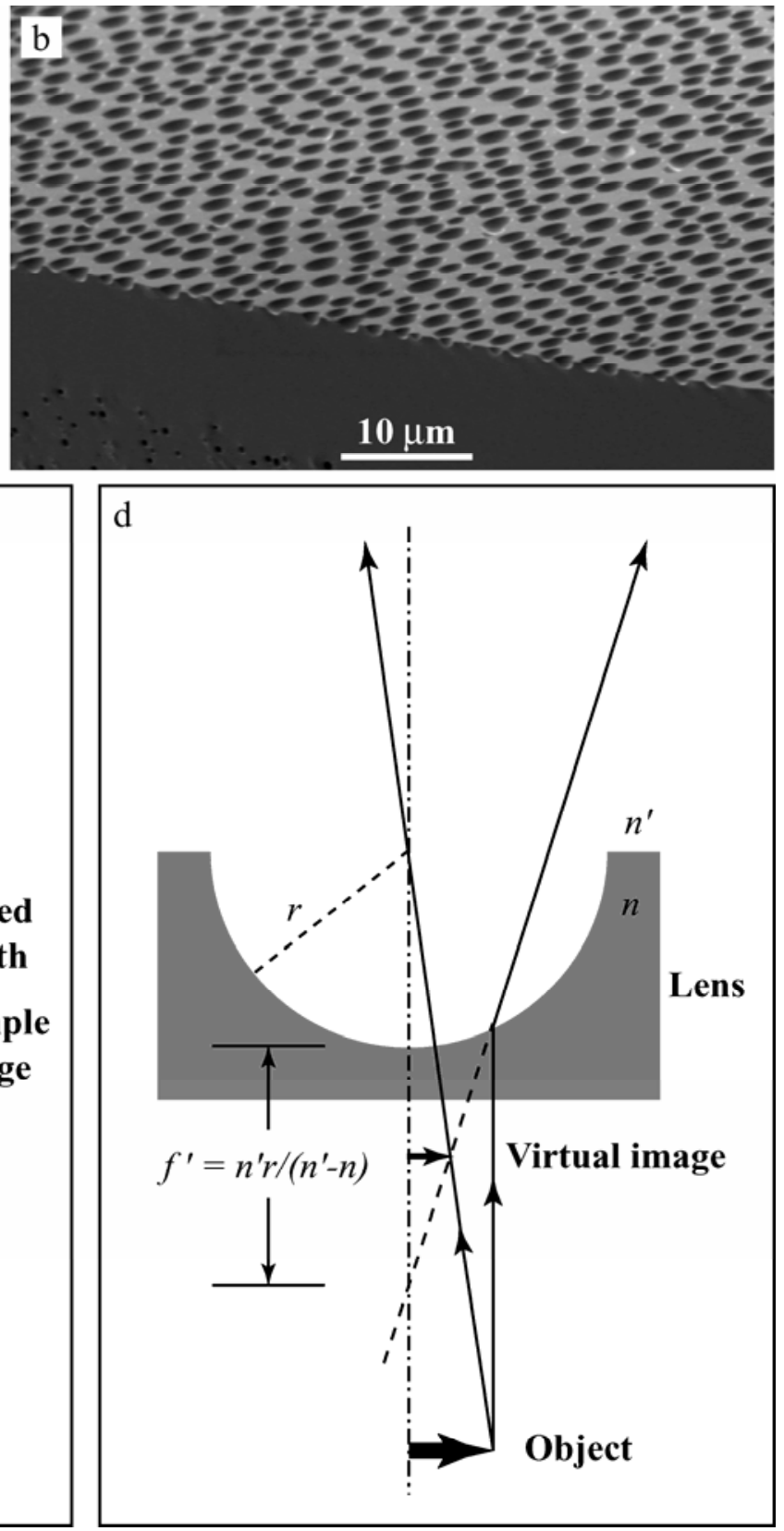

Figure S1. (a) SEM image of concave surface patterns recorded on a non-tilted monolith. (b) SEM image of concave surface patterns recorded on a tilted monolith. (c) Schematic illustration of the optical system for recording virtual image arrays formed with concave surface patterns as microlens arrays. The cross was written on a glass slide. (d) Schematic illustration of the image formation mechanism of an individual concave depression under the paraxial approximation. The refraction of light rays at the air-monolith interfaces is neglected. The objective (Nikon CF Plan) used for recording virtual images formed through concave surface patterns has a magnification of 50 and a 
numerical aperture of 0.45 . Its depth of field is estimated to be $4-5 \mu \mathrm{m}$ (see

http://www.microscopyu.com/articles/formulas/formulasfielddepth.html for information on the depth-of-field values of Nikon objectives). The refractive index of monolithic silica containing L31 copolymers is 1.43 (Yang, P. D.; Wirnsberger, G.; Huang, H. C.; Cordero, S. R.; McGehee, M. D.; Scott, B.; Deng, T.; Whitesides, G. M.; Chmelka, B. F.; Buratto, S. K.; Stucky, G. D. Science 2000, 287, 465-467). The focal lengths of concave lenses are estimated to be $\sim 4.7 \mu \mathrm{m}$, using the refractive index of monolithic silica and the average diameter of concave depressions $(\sim 4 \mu \mathrm{m})$ and assuming each concave depression has a spherical shape. Because the object distance is much larger than the focal length of each concave lens in our experiment, the image distance is close to the focal length. Therefore, the virtual images of the cross and the bottoms of concave depressions were simultaneously observed.

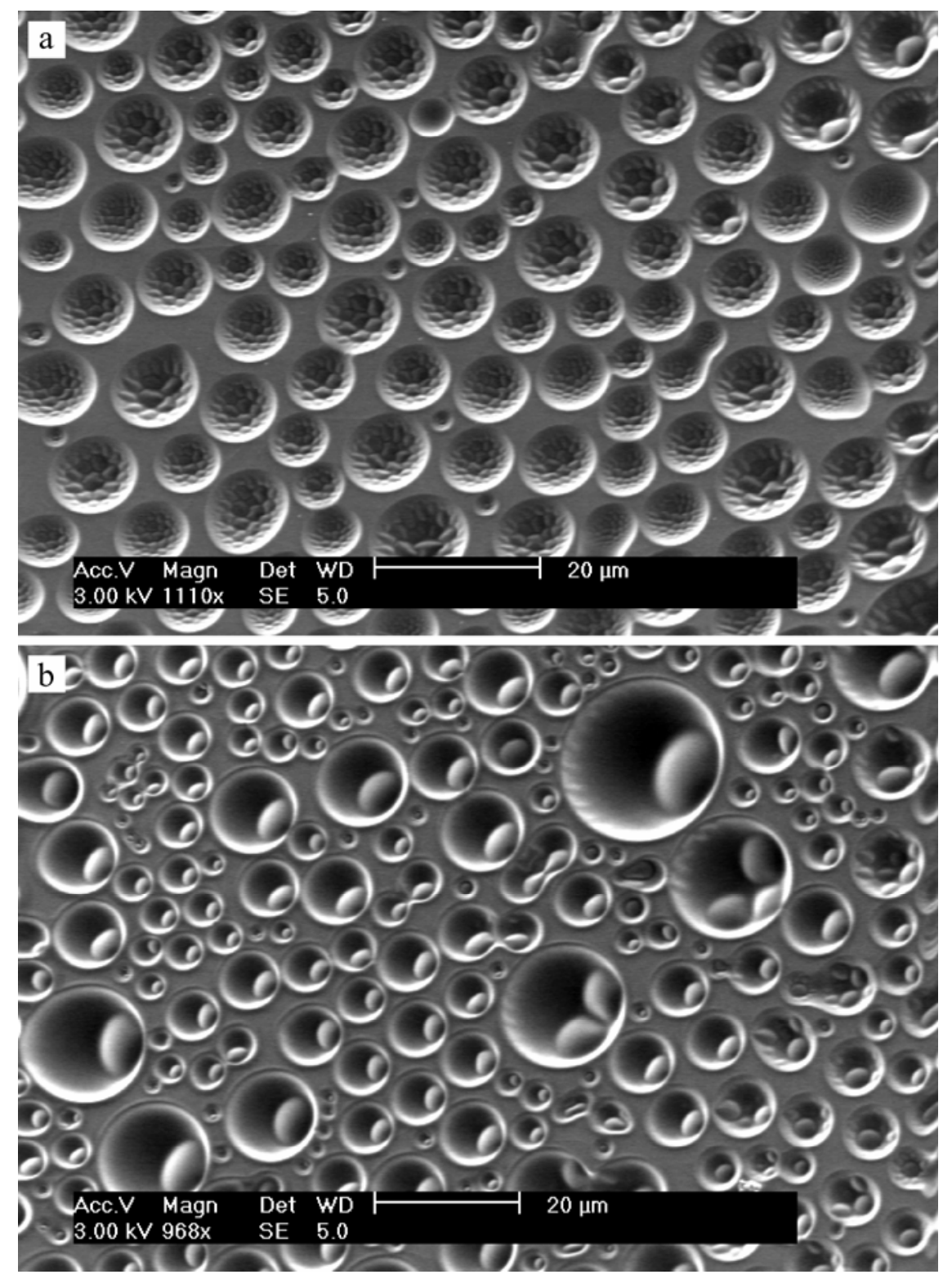

Figure S2. SEM images of hierarchical surface patterns. (a) Each concave depression contains tens of convex protrusions. (b) Each concave depression contains one or a few convex protrusions. 